\title{
Intervening on psychopathology networks: Evaluating intervention targets through simulations
}

\author{
Gabriela Lunansky ${ }^{1 \mathrm{a}}$ \\ Jasper Naberman ${ }^{\mathrm{a}}$ \\ Claudia D. van Borkulo ${ }^{\mathrm{a} b}$ \\ Chen Chen ${ }^{\mathrm{cd}}$ \\ Wang $\mathrm{Li}^{\mathrm{cd}}$ \\ Denny Borsboom ${ }^{\mathrm{a}}$
}

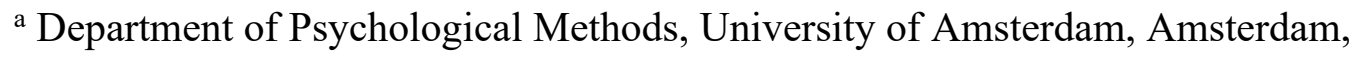
The Netherlands

${ }^{\mathrm{b}}$ Centre for Urban Mental Health, University of Amsterdam, The Netherlands

${ }^{c}$ Laboratory for Traumatic Stress Studies, CAS Key Laboratory of Mental Health, Institute of Psychology, Chinese Academy of Sciences, Beijing, China

${ }^{\mathrm{d}}$ Department of Psychology, University of Chinese Academy of Sciences, Beijing, China

\section{Declaration of Interest}

None.

\footnotetext{
${ }^{1}$ Correspondence concerning this article should be addressed to Gabriela Lunansky, Department of Psychological Methods, University of Amsterdam, Nieuwe Achtergracht 129-B, 1018 WS Amsterdam. E-mail: g.lunansky@uva.nl. Correspondence concerning the presented R-package nodeIdentifyR should be addressed to Jasper Naberman. E-mail: jaspernaberman@live.nl
} 


\begin{abstract}
Identifying the different influences of symptoms in dynamic psychopathology models may hold promise for increasing treatment efficacy in clinical applications. Dynamic psychopathology models study the behavioral patterns of symptom networks, where symptoms mutually enforce each other. Interventions could be tailored to specific symptoms that are most effective at lowering symptom activity or that hinder the further development of psychopathology. Simulating interventions in psychopathology network models fits in a novel tradition where symptom-specific perturbations are used as in silico interventions. Here, we present the NodeIdentify $R$ algorithm (NIRA) to identify the projected most efficient, symptom-specific intervention target in the Ising model. This algorithm is implemented in a novel and freely available $\mathrm{R}$ package. The technique studies the projected effects of symptom-specific interventions by simulating data while symptom parameters (i.e., threshold parameters) are systematically altered. The projected effect of these interventions is defined in terms of the expected change in overall symptom activity across simulations. With this algorithm, it is possible to study (1) whether symptoms differ in their projected influence on the behavior of the symptom network, and, if so, (2) which symptom has the largest projected effect in lowering and increasing overall symptom activation. As an illustration, we apply the algorithm to an empirical dataset containing assessments of PTSD symptoms in a sample that experienced the Wenchuan earthquake in 2008. The most important limitations of the method are discussed, as well as recommendations for future research, such as shifting towards modeling individual processes to validate these types of simulation-based intervention methods.
\end{abstract}

\title{
Keywords:
}

psychopathology, network analysis, dynamic systems, simulation study, interventions 


\section{Introduction}

Recent research focuses on the distinct roles that symptoms may play in the development of psychopathology [1]. For example, some symptoms could have stabilizing effects, meaning that once they are present, they also activate related symptoms (e.g., the presence of the depressive symptom "fatigue" also leads to the activation of the symptom "loss of energy") [2]. In this way, these stabilizing symptoms may influence the spread of symptom activity and the development of psychiatric disorders. Investigating whether symptoms have different roles in the onset and development of psychopathology, and, if so, developing a methodology to identify the most influential symptoms could have promising clinical implications for increasing treatment efficacy [3], [4]. Clinical interventions could be tailored to specific symptoms that are most effective in lowering symptom activity or that hinder the further development of psychopathology.

Treatments for mental disorders already make use of symptom-specific interventions. For example, in the case of Generalized Anxiety Disorder (GAD), interventions exist for distinct type of worrying problems using Cognitive Behavioral Therapy (CBT) [5]. In the case of Major Depressive Disorder (MDD), specific treatment programs have been developed for suicidal behavior [6]. Symptom-specific interventions are also being developed in clinical trials, such as particular CBT for psychosis which focuses on treating hallucinations or delusions [7]. Furthermore, symptom-specific treatments are used in experimental settings, such as randomized controlled trials, to compare the specific effects of different treatment conditions, for example, between psychotherapy and psychopharmacology [8]. By using novel technology, "micro-interventions" can be administered via smartphones as a personalized approach to target the depressed mood symptom [9]. However, it is vital to consider the propelling effects from intervening on one symptom to other symptoms due to their potential interrelatedness [4].

An established framework to study psychopathology as an interrelated, dynamic system of symptoms is the network theory of mental disorders [2], [10]. The network theory of psychopathology has successfully been applied to a variety of psychiatric disorders (e.g., for MDD, see [11], for GAD, see [12], for Post-Traumatic Stress Disorder, see [13], for Psychosis, see [14]; and for Autism Spectrum Disorder, see[15]). According to this theory, symptoms are not passive indicators of one underlying mental disorder but play an active part in developing and maintaining psychopathology. Symptoms are organized in a network structure that encodes unique associations between symptoms [2], [10]. Networks consist of nodes that represent symptoms and edges that represent the associations between symptoms (see Figure 1). 


\section{Figure 1}

\section{Example of a Symptom Network Model}

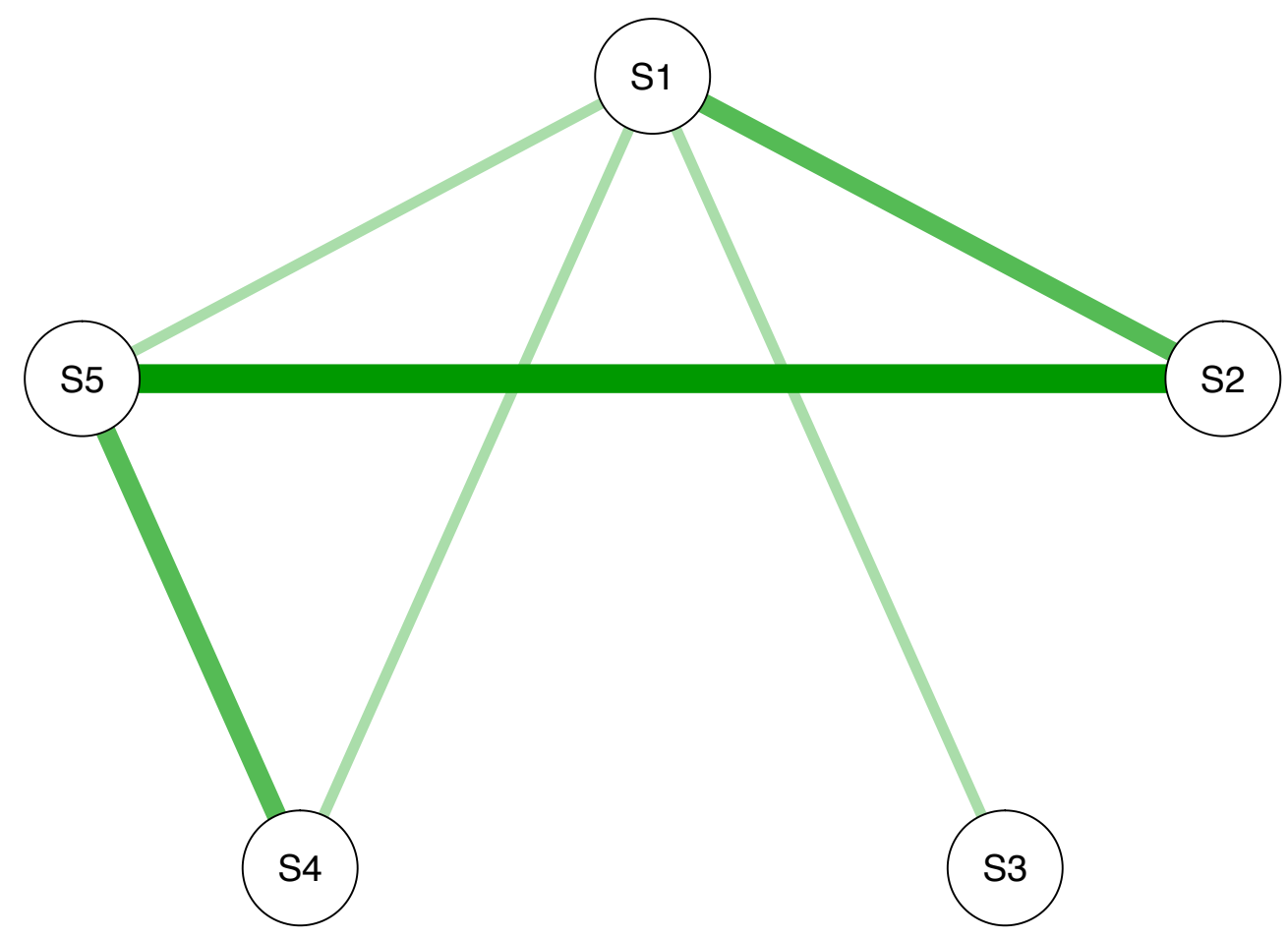

Note. A hypothetical symptom network model for five psychopathology symptoms (S1-S5). Circles in the network represent nodes, which refer to the symptom variables (S1-S5). Lines that connect the circles represent edges, where green (red) lines represent positive (negative) associations. The thickness of the edges represents the magnitude of their association. In this hypothetical network, there is a relatively strong association between S5 and S2, which means that if S2 is activated, S5 is likely to activate as well, and vice versa. Contrary, there is no direct relation between S3 and S4 when controlling for the other nodes in the network (S1, S2, and S5).

Psychopathology network models analyze the co-occurrence of symptoms estimated from data, such as clinical interviews or questionnaires [17]. In general, these models consist of two types of parameters: parameters for nodes and parameters for edges [18], [19]. Edge parameters are called edge weight parameters, and denote the unique, weighted, statistical associations between a pair of symptoms when controlling for the presence of all other symptoms in the network [20]. Positive (negative) edge weight parameters denote positive (negative) associations. For example, suppose two symptoms such as "worry" and "irritability" are strongly positively associated. In that case, the theory proposes the hypothesis that the presence of the "worry" symptom lead to the activation of the "irritability" symptom as well, and vice versa [2], [10]. In network models estimated from continuous data, such as the Gaussian Graphical Model (GGM) [21], edge weights are computed from the partial correlations of each 
pair of nodes. In network models estimated from discrete data, such as the Ising model, edges are calculated based on logistic regression analyses, in which variables are iteratively regressed on all other variables except the variable itself [18], [22]. To obtain sparsity and account for false-positive edges, regularization is imposed on the network structure, meaning that small edge parameters are shrunk to zero [22].

Parameters for the nodes differ across network models, depending on the type of data from which they are estimated. For networks fitted to discrete data, node parameters are called threshold parameters and denote the weighted internal dispositions for the activation of each symptom [18], [22]. The threshold parameter is calculated as the intercept of the logistic regression equation when the variable is regressed on all other variables [22]. Thus, every variable in the network has its threshold parameter, where a positive (negative) threshold denotes the node's predisposition to be activated (deactivated) if all other symptoms are absent ${ }^{2}$ [22]. The larger the value of the threshold parameter, the larger the probability that a symptom will be activated [18].

To assess the relative importance of symptoms in psychopathology networks estimated from observational data, the concept of node centrality was received with high hopes [23]. Centrality indices stem from the domain of social networks, in which the most central node in the network has the largest number of edges with neighboring nodes and the most substantial edges [24]. The concept was translated to psychology [25], where the centrality hypothesis states that the most central nodes are the best intervention targets, as they are thought to represent the most influential nodes in a network [26]. Therefore, centrality metrices are used in psychopathology networks to identify possible intervention targets [2], [20], [23], [27], [28]. However, several researchers have raised doubts regarding the suitability of centrality indices in psychological networks [23], [29]-[33]. Centrality indices are based on the structure of the psychological network (i.e., the presence and strength of edges), but do not explicitly consider the dynamics of the network. It is not evident how the structure of statistical network models relates to causal influences of symptoms: a causal process running over the network structure needs to be assumed before one can assess causal claims [30], [34], [35].

A developing novel tradition studies the projected influences of symptoms in psychopathology models using simulated symptom-specific perturbations as in silico interventions [32], [34], [36], [37]. By altering characteristics of the symptom network, such as systematically deactivating symptoms (i.e., altering the symptom variables' state), and simulating data, the symptom's projected influence on the behavior of the network can be studied [32], [34], [36]. For example, the value of a symptom such as "loss of energy" is set to zero to simulate its treatment effect on the rest of the network. The procedure is repeated for all other symptoms in the network. The projected impact of this symptom-specific intervention is calculated as the change in the overall symptom sum score. The node with the most significant expected

\footnotetext{
${ }^{2}$ Depending on the specific model used, and the possible values of the nodes, the threshold parameter could also take a value between 0 and 1 , where 0.5 indicates no preference, 0 indicates a preference for deactivation and 1 indicates a preference for activation. See [66] for an extensive discussion.
} 
influence is the node that propels the most substantial change in the next simulation iteration [36].

However, the clinical representation of simulating an intervention by altering the symptom's state (i.e., forcing the symptom to be absent) does not take into account that nodes all have different thresholds for activation. Interventions differ in their effectiveness to treat symptoms [38]. Furthermore, from a clinical perspective, it is unlikely that a treatment intervention will forever push the presence of a symptom to zero. Instead, interventions are more likely to lower the probability of symptoms being present. In other words, symptoms may still be present from time to time, but after the intervention they are less likely to occur. Therefore, a better clinical representation of simulating interventions would be the alteration of node parameters in a network model.

Symptom threshold parameters can be altered in two ways: by increasing or decreasing nodes' internal dispositions for activation. A symptom's threshold for activation can be decreased so that it is less likely to be activated. This would mimic a clinical intervention on a specific symptom, which we call an alleviating intervention. When done systematically, one can study which alleviating intervention on a specific symptom in a network model has the most substantial projected effect on lowering overall symptom activity. Contrary, a symptom's threshold parameter can also be increased such that it is more prone to activation, which we call an aggravating intervention. This would mimic the effect of a stressful event on the symptom, increasing its probability for activation. Aggravating interventions are used to study which symptom would have the most substantial projected effect on deteriorating the network's state in a stressful event.

This paper presents an algorithm that outlines node-specific target points for interventions on psychopathology networks, which are estimated from observational data. This algorithm focuses on the clinical importance of a node by altering its threshold parameter and studying its projected effect on the behavior of the network. With this algorithm, it is possible to study (1) whether symptoms have distinct projected influences on the behavior of the network, and if so, (2) which symptom has the most substantial projected effect after an alleviating intervention and aggravating intervention. In the following section, the algorithm is explained and applied to an empirical dataset containing assessments of Post-Traumatic Stress Disorder (PTSD) symptoms.

\section{Methods}

In this section, we explain the rationale behind the proposed technique. Furthermore, we outline the analysis design in which we apply the technique to an empirical dataset of PTSD symptoms. 


\subsection{NodeIdentifyR algorithm}

We present the NodeIdentifyR algorithm (NIRA) to identify the projected most efficient, symptom-specific intervention target in psychological networks ${ }^{3}$. This technique studies the projected effects of symptom-specific interventions by simulating data when symptom parameters are systematically altered. The effect of these perturbations is calculated as the change in overall symptom activation of the network.

\subsubsection{Model}

The algorithm uses the Ising Model as a representation of psychopathological dynamic systems. The Ising model originates in physics and describes the interaction between states of particles that are connected in a network (originally, the Ising model was constructed to explain magnetism; [39]. Since the model's characteristics align with the network theory of psychopathology, it is often used as a statistical model of symptom networks [18], [40]. The model is sufficiently simple to be mathematically tractable, and at the same time sufficiently rich to represent important phenomena of mental disorders. For example, the presence of alternative stable states (i.e., the system can be in a healthy state or disorder state), critical transitions (i.e., the system can suddenly jump towards a disordered state when faced with enough stress) and hysteresis (i.e., once the system is stuck in the disordered state, it requires a stronger reduction of stress to recover than the original level of stress that caused the critical transition; [11]. The Ising model uses binary data, and in the current study, responses are coded as either 0 or 1 , indicating the symptoms' absence or presence.

\subsubsection{Interventions}

All analyses and simulations are executed using the statistical software program $R$. NIRA runs multiple simulations, one with all the original threshold parameter values and one for every individually altered threshold parameter. To be precise, NIRA will generate 5000 observations or simulated 'participants' for which symptoms are assessed. Every variable here maps to a specific symptom in the network. For example, in a network containing ten symptoms, $11 \mathrm{x}$ 5000 observations will be generated: once with all original threshold parameter values and ten times where a different threshold parameter is changed iteratively. Response simulations are computed with the R package IsingSampler [41], which samples states from the probability distribution of the Ising model. NIRA uses the Metropolis-Hastings algorithm implemented in IsingSampler for data generation, to ensure the process will remain computationally feasible in a multivariate distribution [41]. Note that the Metropolis-Hastings algorithm does not return the exact likelihood, but a pseudo-likelihood; the exact likelihood can be computed for small networks (up to $\sim 10$ nodes) with the function IsingLikelihood, but this is infeasible for larger networks due to the intractability of the Ising model [41].

The magnitude of the intervention, specifically, the value with which the threshold parameters are increased or decreased, determines the strength of the intervention on the network's

\footnotetext{
${ }^{3}$ The nodeIdentifyR R-package can be downloaded via: https://github.com/JasperNaberman/nodeIdentifyR Note that the R-package is in a development stage. Correspondence concerning the presented R-package nodeIdentifyR should be addressed to the main developer Jasper Naberman. E-mail: jaspernaberman@live.nl
} 
behavior, thus, the intervention's effect size. In the current study, NIRA determines the standard deviation of the distribution of all original threshold parameters (which are in turn estimated from empirical data) and alters the current value of the threshold parameter in question with two times that standard deviation. In this way, the magnitude of the intervention is somewhat bound to the distribution of the thresholds. A potential downside is that the magnitude of the intervention depends on the distribution of the raw data, and changes over different datasets. However, chosing a fixed magnitude (e.g., subtracting or adding a value of one to the thresholds) is suboptimal since its effect size will also change depending on the original value of the estimated threshold parameters. Appendix A shows a sensitivity analysis when adjusting the magnitude of the intervention to one standard deviation of the threshold's distribution. In the R-package, the magnitude of the intervention can be adjusted to the number of standard deviations of choice.

Furthermore, it is important to note that when simulations are used to study projected effects, the simulated behavior of the model needs to converge to a stable state to ensure results are robust (see, e.g., [42]). Multiple iterations are necessary to ensure that the simulated behavior is robust and replicable [43]. Therefore, we will simulate the effect of interventions on the behavior of the symptom networks until the model has converged to a stable state (See Appendix B for stability analyses of NIRA using various numbers of iterations).

Two types of interventions can be administered. Alleviating interventions decrease a symptom's threshold by subtracting two standard deviations of the thresholds' distribution from its original value. In contrast, aggravating interventions increase a symptom's threshold by adding two standard deviations to its original value.

\subsubsection{Determining the most effective target}

To study the projected effect of an intervention on the entire network, sum scores are inspected. The sum score of a simulated observation equals the sum of all data points for that observation. Since the Ising model uses binary data, responses are decoded as either 0 or 1 , indicating the symptom's absence or presence. In an exemplary questionnaire consisting of ten items, the sum score of each observation can range from zero to ten. Higher scores would indicate higher levels of psychopathology. The use of sum score analyses in a simulation environment to measure the impact of specific perturbations can be used effectively to measure the overall state of a dynamic system [44].

The NIRA outcome will be computed as the absolute difference between the baseline network's sum score (without interventions) and the sum scores after every threshold alteration, for both alleviating and aggravating interventions. The node-specific intervention with the highest absolute difference is the node with the strongest projected effect on the network's behavior.

\subsection{An empirical application to PTSD}

As an empirical illustration, NIRA is applied to a dataset containing PTSD symptoms. Three research questions are investigated: (1) Do symptoms differ in their projected influences on the network's behavior after node-specific interventions? (2) Are identical nodes identified by 
NIRA for alleviating interventions and aggravating interventions? (3) Is the most efficient target node identified by NIRA also the most central node?

\subsubsection{Data}

The empirical dataset contains PTSD symptom assessments, gathered after the 2008 Wenchuan earthquake. The sample consisted of 4910 adolescents ( $49.5 \%$ boys; mean age $11.4 \pm 1.4$ years) who experienced the earthquake and was measured 2.5 years after the earthquake. Their 17 DSM-IV PTSD symptoms were assessed by the Chinese version of the University of California, Los Angeles PTSD Reaction Index (PTSD-RI; [45]), a validated self-rated 5-point Likert scale (from $0=$ never to $4=$ most of the time). Missing item responses were handled using full information maximum likelihood (ML) procedures. To estimate the Ising models, we binarized the symptom scores into 0 (original score was 0 ) and 1 (original score ranged from 1 to 4), respectively, representing symptom absence and (at least some level of) symptom presence.

\subsubsection{Design}

NIRA uses the IsingFit package [40] to estimate the Ising Models and the qgraph package [17] to visualize the networks. NIRA is applied twice to the network: once with alleviating interventions and once with aggravating interventions. To study the relationship between the size of the original threshold values and the ordering of the projected most effective intervention targets, the correlation between the novel threshold values after interventions and the NIRA outcome will be computed.

\subsubsection{Comparison with strength centrality}

Node centrality indices, precisely, strength centrality are calculated for the Ising model using the qraph package [17]. Strength centrality is defined as the sum of the absolute weighted edge strengths, where the sum is taken over edges that are connected to the relevant node [25]. Nodes with higher strength centrality have more and stronger connections with neighboring nodes and are therefore often hypothesized to be more influential in the spread of symptom activity [33]. Stability studies have shown that strength centrality is the most robust centrality measure used in psychological networks, especially in ordering symptoms [20]. The correlation between strength centrality and NIRA will be computed.

\section{Results}

Figure 2 shows the estimated Ising model network from the PTSD symptoms. Nodes in the networks represent 17 PTSD symptoms from three subdomains: Intrusion, Avoidance, and Arousal (see Table 1). 


\section{Figure 2}

Ising Model of the PTSD Symptoms

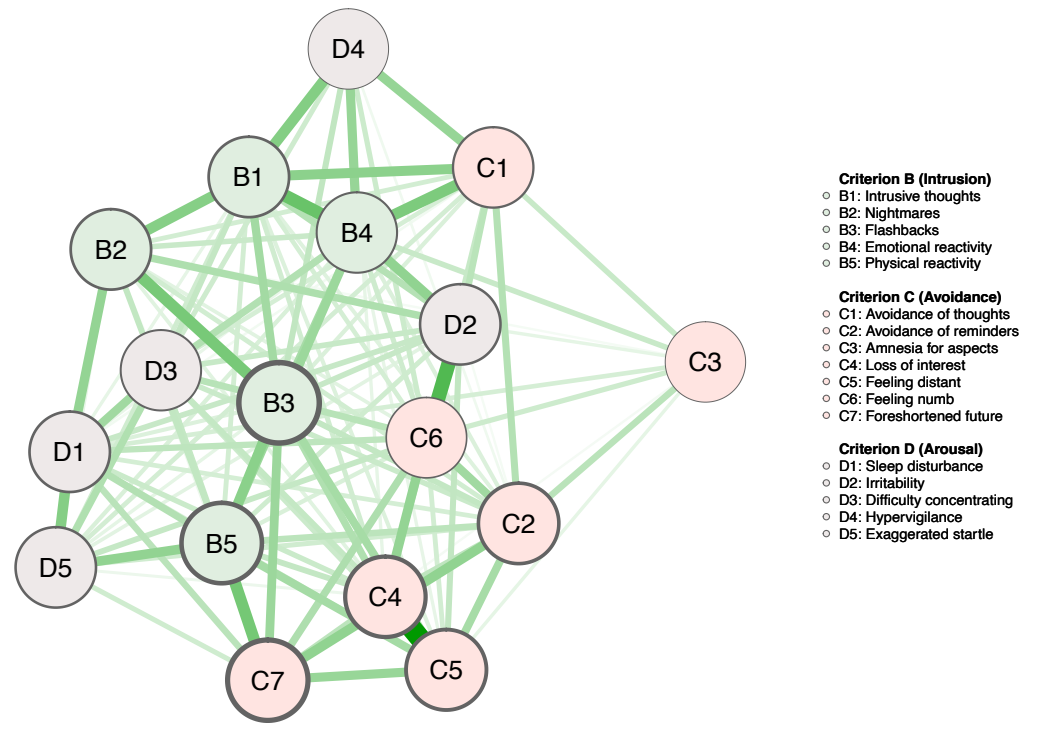

Note. Nodes in the networks represent the 17 PTSD symptoms. Symptoms are grouped by color based on their clinical subdomain (Intrusion, Avoidance, and Arousal). The thickness of node borders represents the absolute value of the nodes' threshold parameters (all thresholds are negative, except the threshold of node "D1").

[INSERT TABLE 1 HERE]

\subsection{Interventions}

\subsubsection{Projected effects of alleviating interventions}

First, NIRA was applied to the Ising model using alleviating interventions (see Figure 3). Results show that symptoms have different projected influences on the network's behavior when targeted with alleviating interventions. For example, symptom B1 (Intrusive thoughts) lowers the projected symptom sum score from 10.77 to 8.83 . Contrary, symptom C7 (Foreshortened future) merely lowers the projected sum score to 10.01. These results suggest that symptoms may have propelling effects on the decrease of PTSD levels. Instead of lowering the overall sum score by one point when intervening on one symptom, symptom B1 is projected to lower the sum score by two points after an alleviating intervention. Thus, according to NIRA, intervening on B1 could have propelling effect on PTSD levels.

The correlation between the threshold parameter magnitudes after alleviating interventions and NIRA is $r=-0.34$ (see Figure 4). This indicates a moderate relationship between the threshold values and NIRA outcomes, however, threshold values in isolation cannot fully explain the 
results from NIRA. In other words, projected effects from symptom-specific NIRA interventions also depend on the edge parameters in the network.

\section{Figure 3}

\section{Projected Effects of Alleviating Interventions}

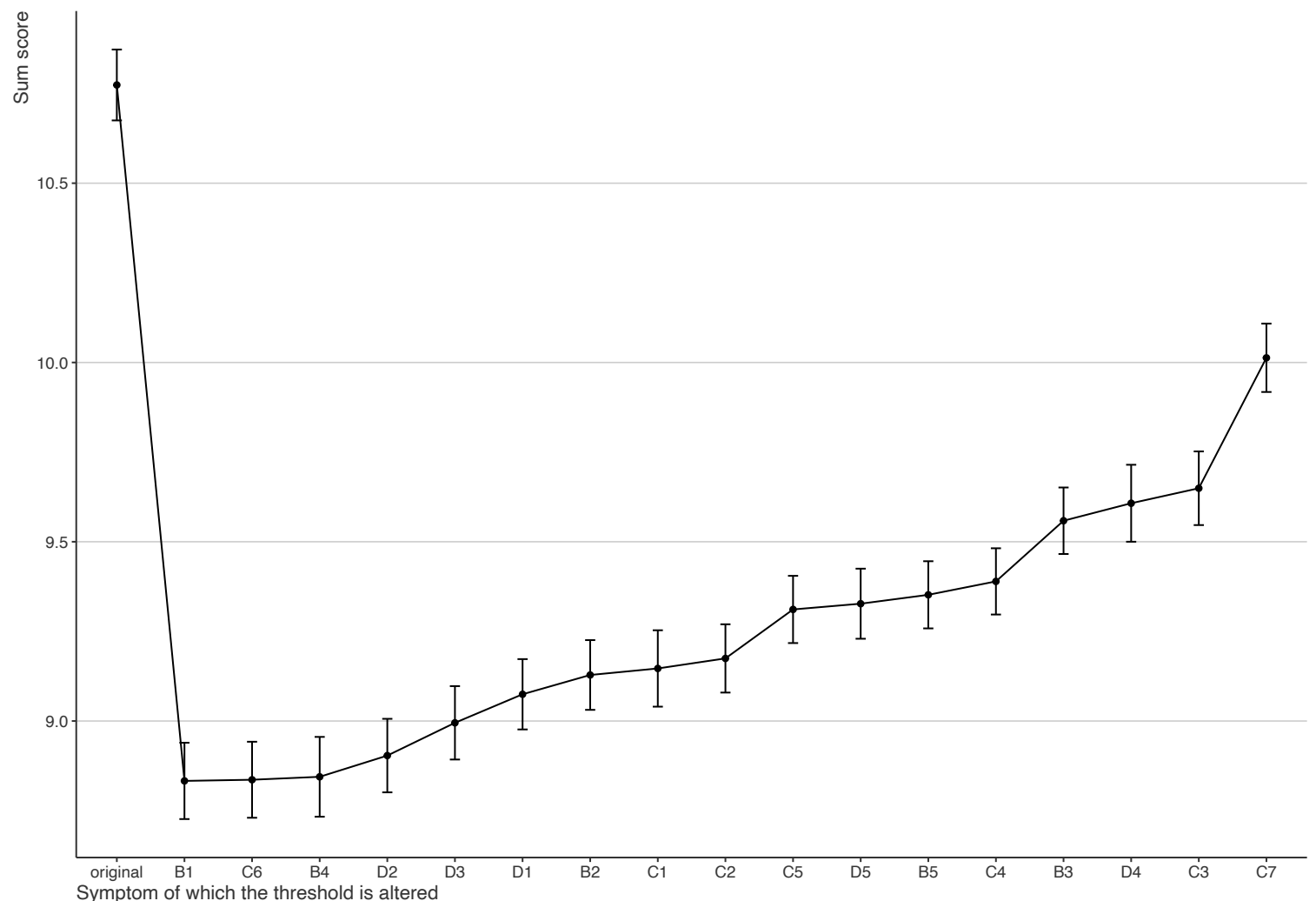

Note. Results after alleviating interventions from NIRA to the PTSD Ising Model. The Xaxis shows the symptoms of which the threshold is altered, including the original projected sum score of active symptoms, i.e., when data are simulated from the network without altering threshold parameters. Afterwards, the projected effects on decreasing the sum score are shown when data are simulated after altering the thresholds of one node at a time. The black lines show the results on the mean symptom sum score after every intervention, including the confidence intervals. 


\section{Figure 4}

The Relation between Thresholds and NIRA Alleviating Interventions

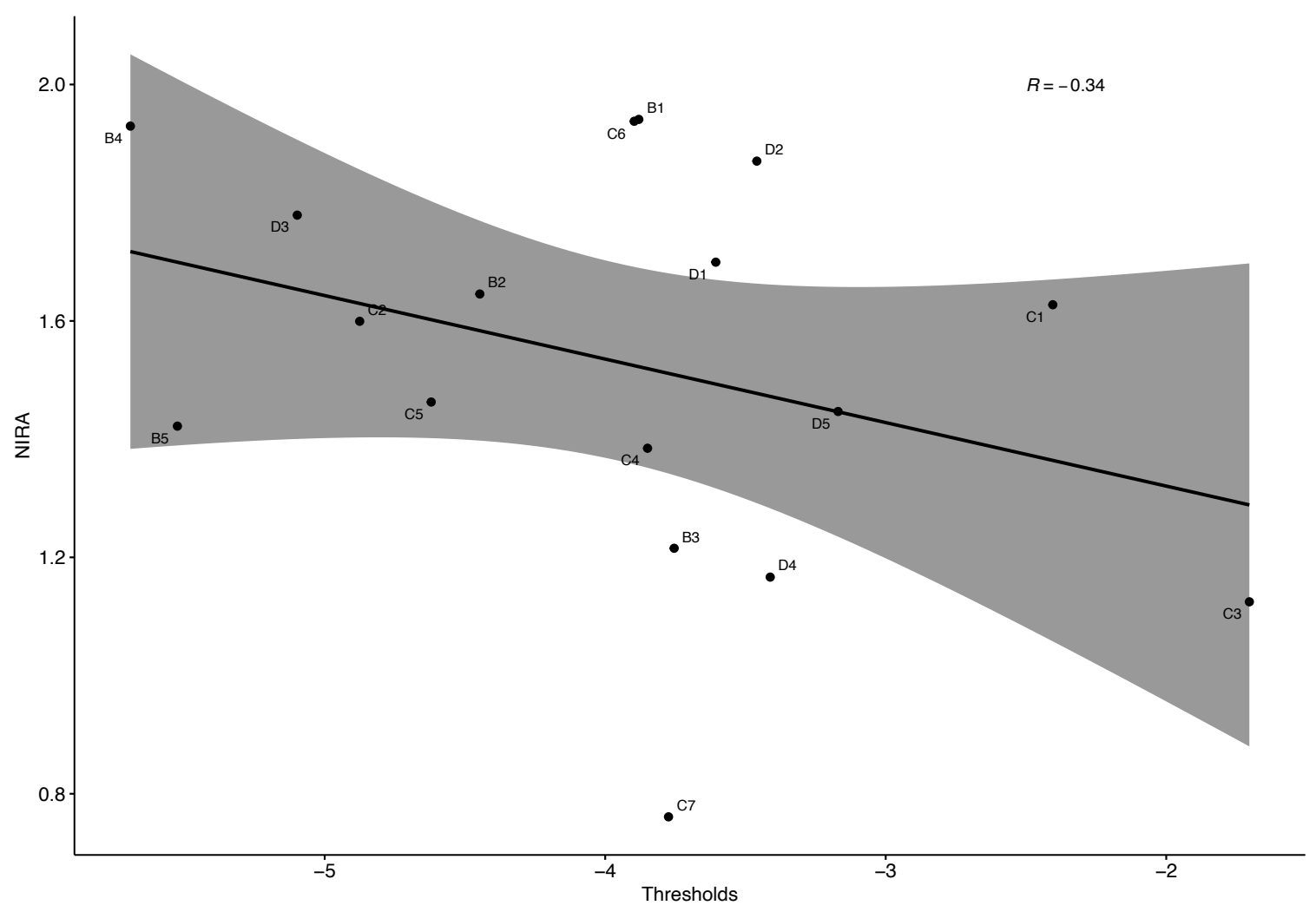

Note. The $\mathrm{x}$-axis shows the magnitude of the threshold parameters after alleviating interventions. The y-axis shows the NIRA coutome, computed as the absolute difference between the original sum score of the network and after each intervention. The grey area represents the $95 \%$ confidence interval. The correlation of $r=-0.34$ indicates that there is a moderate relationship between the distribution of the threshold magnitudes and their projected effect according to NIRA.

\subsubsection{Projected effects of aggravating interventions}

Second, we applied aggravating interventions to the Ising model using NIRA (see Figure 5). The results show that symptoms have different projected influences after aggravating interventions. For example, the symptom C7 (Foreshortened future) has the strongest projected effect on increasing the sum score (from 10.77 to 12.53). In contrast, symptom D4 (Hypervigilance) has the lowest projected effect (increasing the sum score to 11.05). Therefore, the results suggest the presence of propelling effects when the network is faced with aggravating interventions.

The correlation between the magnitude of the thresholds after aggravating interventions and NIRA outcomes is $r=-0.31$ (see Figure 6). This correlation indicates that there is a moderate 
relation between the threshold distribution and the ordering of nodes based on their projected effects from NIRA aggravating interventions. In other words, the network's edges also influence the projected effects of node-specific interventions.

\section{Figure 5}

Projected Effects of Aggravating Interventions

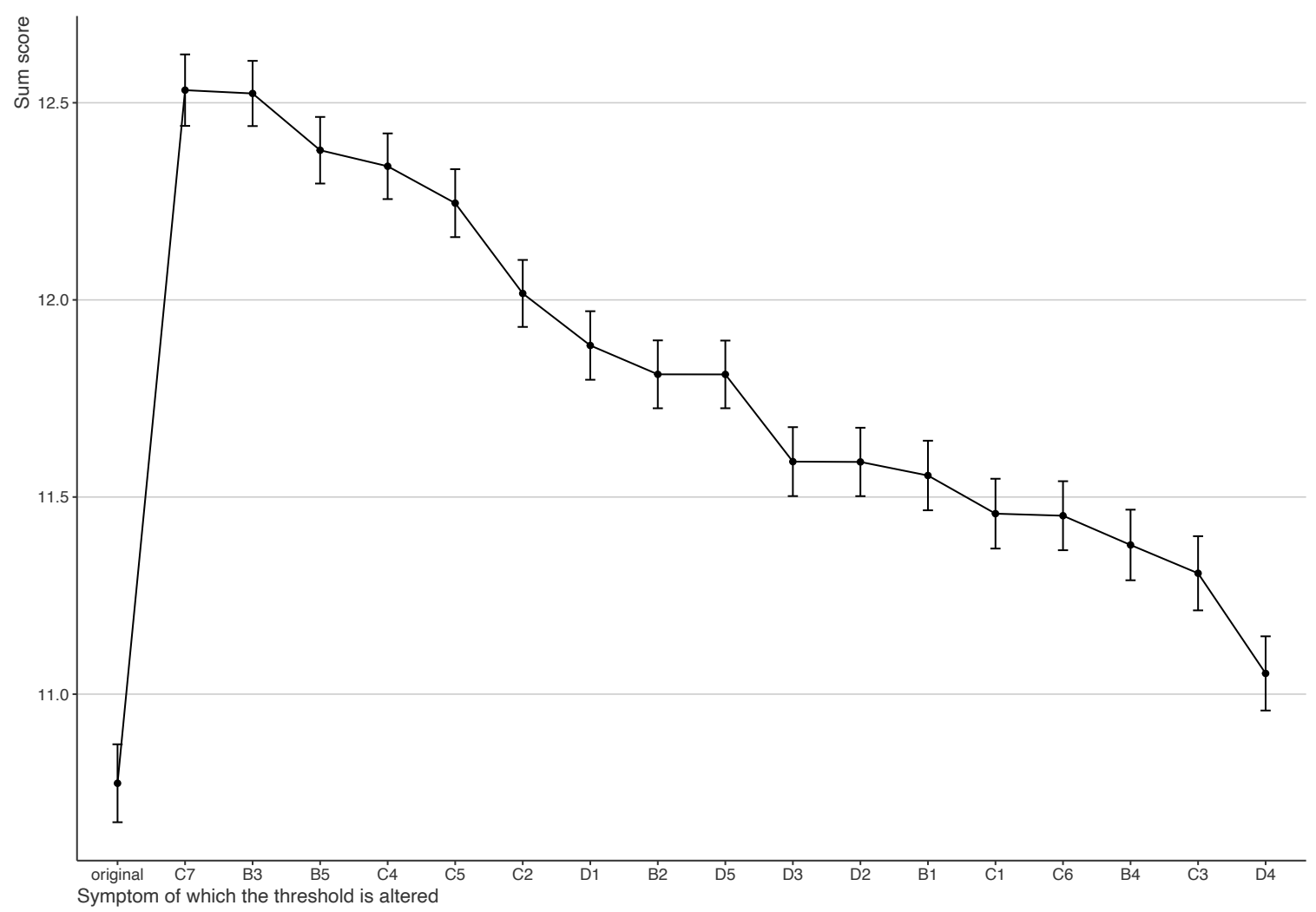

Note. Results from targeting the PTSD Ising Model with aggravating interventions. The original condition shows the projected sum score without altering any threshold parameters. Afterwards, threshold parameters are altered iteratively and data are simulated to calculate the interventions' effects on the behavior of the network. Mean symptom sum scores are shown in black, including confidence intervals. 


\section{PREPRINT WITHOUT PEER-REVIEW}

\section{Figure 6}

The Relation between Thresholds and NIRA Aggravating Interventions

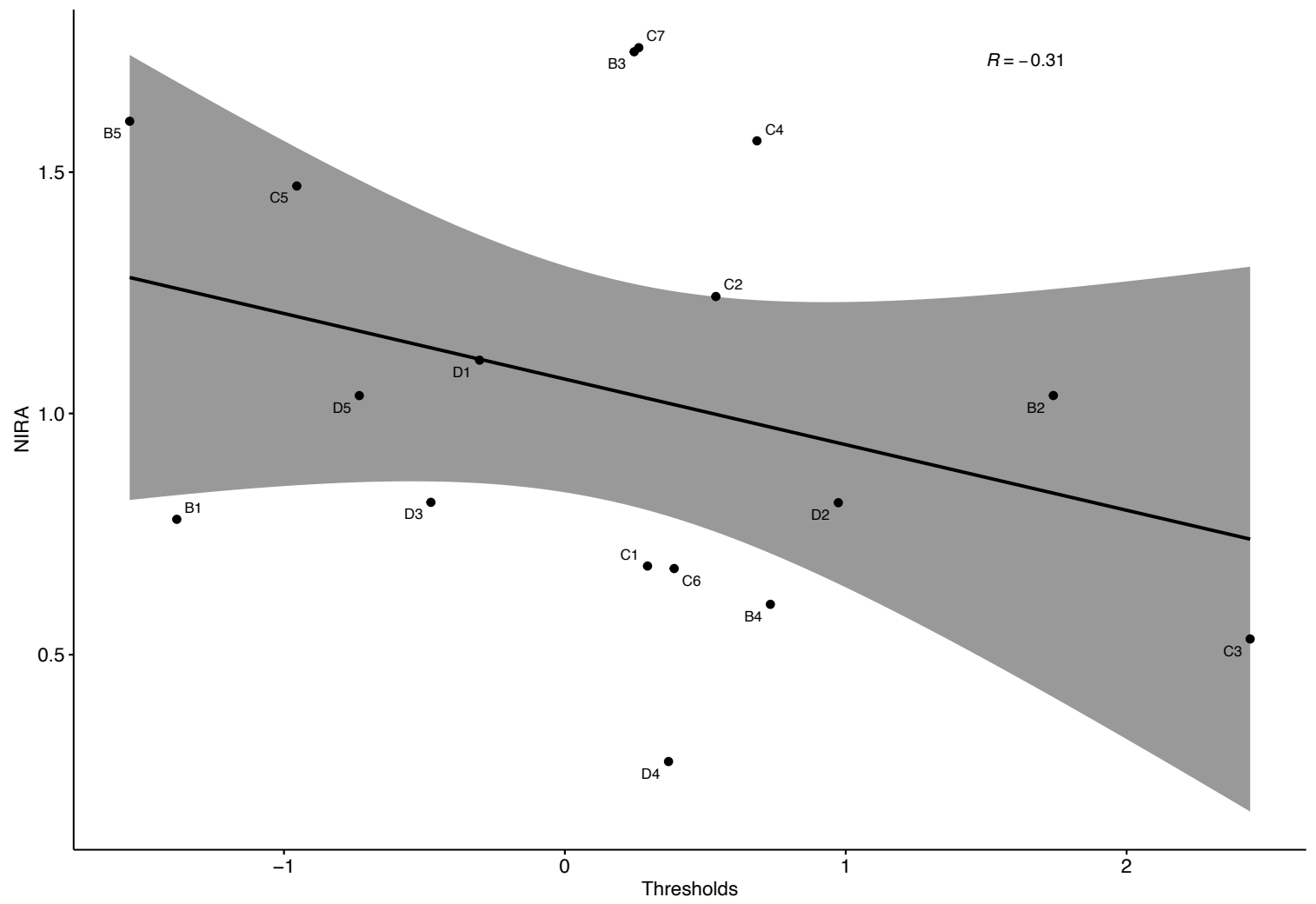

Note. The $\mathrm{x}$-axis shows the threshold values of every node after aggravating interventions from NIRA. The y-axis shows the absolute difference between the network's original sum score and after each node-specific intervention. The grey area indicates the $95 \%$ confidence interval. The correlation of $r=-0.31$ indicates a moderate relation between the projected effects of aggravating NIRA interventions and the distribution of the nodes' threshold parameters.

\subsubsection{Comparing alleviating interventions and aggravating interventions}

To evaluate whether nodes can have different roles in the spread or hinder of symptom activity, we compared the results between alleviating and aggravating interventions (see Figure 7). The results in Figure 7 are ordered based on the projected effects from alleviating interventions. Results suggest that alleviating and aggravating interventions have different effects on the same nodes. For example, symptom B1 (Intrusive thoughts) is projected to be the most effective target for clinical interventions, as it has the largest projected effect in lowering PTSD levels after alleviating interventions. However, it is not the projected most effective target for preventive care, as the network's behavior is not heavily affected by a aggravating intervention on B1. 


\section{Figure 7}

Comparing Alleviating and Aggravating Interventions from NIRA

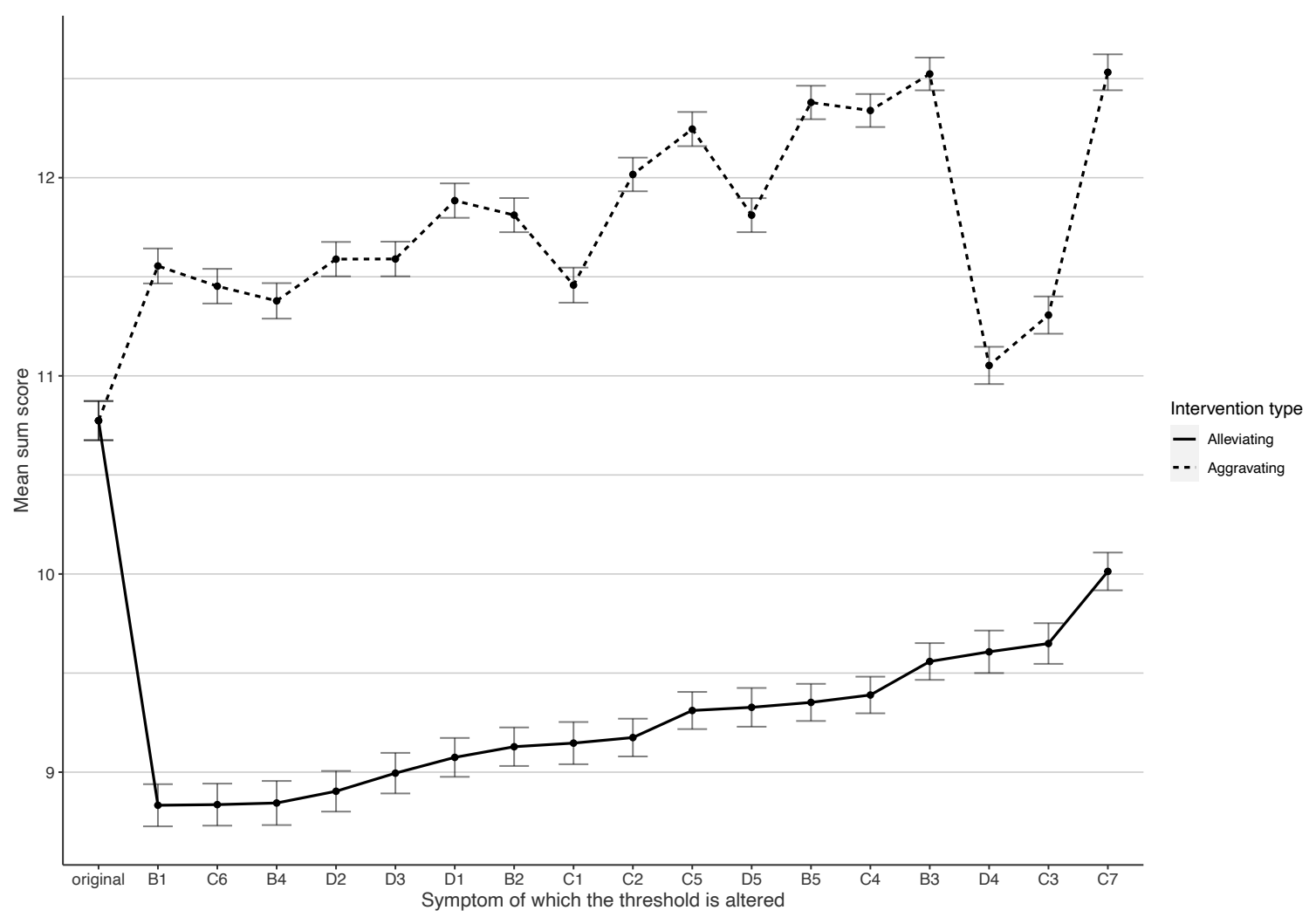

Note. Projected results after alleviating interventions and aggravating interventions. The xaxis shows the PTSD symptoms of which the threshold is altered in the order of projected effects for alleviating interventions. The y-axis shows the symptom sum scores of the network after every symptom-specific intervention. The solid lines represent results from alleviating interventions and the dashed lines the results from aggravating interventions.

\subsubsection{Comparing strength centrality and NIRA}

Figure 8 shows the results from comparing node strength centrality with alleviating and aggravating interventions from NIRA. The correlation between alleviating interventions from NIRA and strength centrality is $r=0.51$, and between aggravating interventions from NIRA and strength centrality is $r=0.43$. Table 2 shows all results, including the ordering of PTSD symptoms based on their strength centrality and projected effects from NIRA interventions. These results indicate a moderate to strong relation between NIRA outcomes and strength centrality. 


\section{Figure 8}

Comparing Strength Centrality with Interventions from NIRA

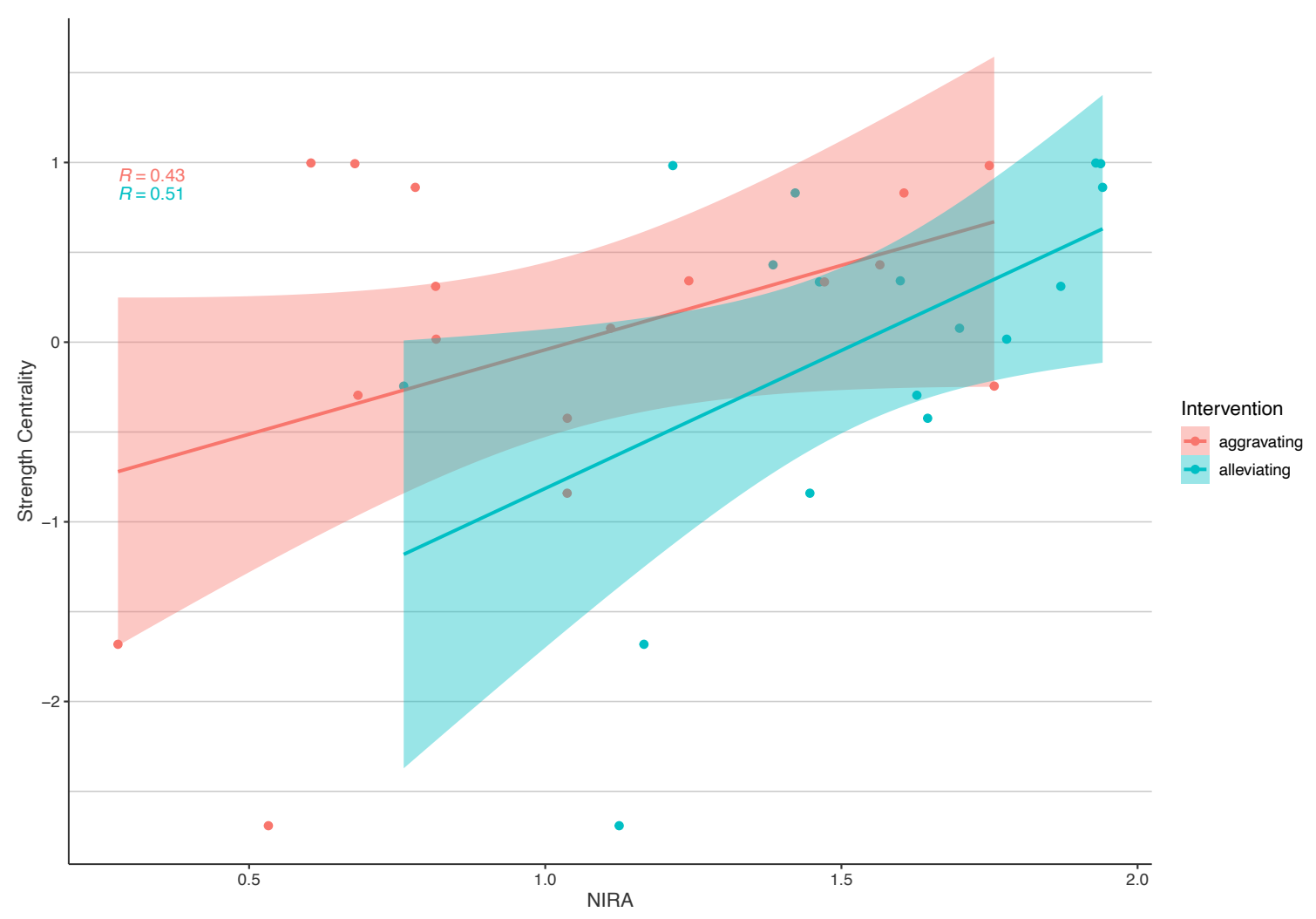

Note. The relation between node strength centrality and projected effects from NIRA interventions. The blue dots and lines represent results from alleviating interventions, and red dots and lines the results from aggravating interventions. The area around the lines represents the $95 \%$ confidence interval.

\section{[INSERT TABLE 2 HERE]}

\section{Discussion}

In this paper, we presented a straightforward, simulation-based technique to study the projected effects of symptom-specific interventions on the overall behavior of psychopathology networks. As an empirical illustration, we applied the technique to an empirical dataset containing PTSD symptom assessments.

NIRA focuses on the clinical relevance of interventions by studying the projected propelling effect of a symptom-specific intervention on the behavior of the network as a whole. The technique can be used to study the projected effectiveness of different symptom-specific interventions. By altering node parameters instead of node states, NIRA aims to better represent the clinical practice where symptom interventions aim to lower the symptom's activation probability. Furthermore, NIRA distinguishes between alleviating and aggravating interventions. The former interventions could be helpful to determine which symptom may be 
the most effective target for clinical interventions, the latter to consider which node symptom may be taken into account for preventive care.

As an empirical illustration, we applied the technique to a dataset containing repeated measures of 17 PTSD symptoms in a sample of participants that experienced the Wenchuan earthquake in 2008. We estimated an Ising model and applied NIRA. Results show that symptoms have different projected influences on the behavior of the network after interventions. These results support the idea that some symptoms have a different effect on the course of psychopathology than others [2], [10], [11]. In the current dataset, symptoms may have propelling effects on lowering or increasing the network's overall symptom activity levels. If there were no propelling effects, intervening on one symptom would change the sum score with a maximum of one point. However, we found that, for example, symptom B1 (Intrusive thoughts) is projected to lower the sum score by two points after an alleviating intervention in period 1 . Important to note is that effect sizes from NIRA depend on the magnitude of the interventions, meaning that propelling effects may change when a smaller intervention strength is chosen (see Appendix A).

Furthermore, we compared results from centrality analyses using the strength centrality index with results from NIRA in the empirical illustration. We found moderate to large correlations, meaning the most effective targets according to NIRA are related to but may differ from the most central nodes. However, more research is needed for more conclusive results, ideally including more types of centrality values (e.g., eigenvector centrality, a metric that takes into account the number of edges of neighboring nodes, and might therefore detect possible propelling effects; [46]).

The presented technique fits into a novel developing tradition that uses simulations for intervention studies in psychological networks [32], [34], [36], [37]. However, this technique has several limitations, of which we will discuss the most pressing ones. The first limitation is that the current version of NIRA can only be used with the Ising model [39]. This means that binary data need to be at hand or data need to be binarized. However, the same logic could be applied to other network models that handle ordinal or Gaussian data, such as the MGM [47] or GGM [21]. For this, the optimal method to alter node parameters in different models needs to be investigated. Further research could develop equivalent techniques like the one presented here for other network models.

Furthermore, there are several limitations regarding the empirical validity of the presented method. One essential feature of the presented technique is that all projected effects depend on the assumption that psychopathology behaves in line with the ferromagnetic Ising model [39]. This is, of course, almost certainly false. It is possible that psychopathology does not behave as a complex system or that current statistical network models, such as the Ising model, do not truthfully represent the complexity of psychopathology. Instead of applying an existing statistical model to psychopathology, one could also try to develop formal models bottom-up, aimed to explain psychological phenomena [35], [37], [48]-[52] or psychological capacities [53]. Using the Ising model to simulate the projected influences of interventions, NIRA remains 
a theoretical exercise, like any other simulation study. Simulation studies teach us what to expect if the used model is the true data-generating model [49]. Thus, the problem of the technique's empirical validity is not limited to the presented method. An advantage of these theoretical exercises, such as simulation-based intervention studies, is that they help generate clear hypotheses that can be tested and falsified in an empirical setting [50]. To clinically validate the projected effects of NIRA, experiments need to be done to test whether (clinical) interventions on the targeted symptoms affect symptom levels as projected.

An issue that further complicates matters is the wide-known fact that it is implausible for population effects to translate to individual processes. In other words, the most effective symptom-specific intervention target to lower the population mean of PTSD is not necessarily the most effective target in individuals due to the heterogeneity of psychological processes [54]-[56]. Ideally, simulation-based idiographic approaches would exist to investigate the most effective intervention target for a specific individual, based on his or her trajectory. One option is using Vector Auto-Regressive (VAR) models [56]. These multilevel models are estimated from intensive longitudinal data (e.g., five measurement moments per day for every participant) and regress all symptom variables on their former measurement moment. One possibility to study the effect of interventions in these VAR models is by using impulse response functions (IRF), where the system receives an external simulated "shock", or impulse, to study its response over time [57], [58]. IRF is used in economics (see, for example, [59]), and we hope that psychological research will further expand into that direction. However, until these methods are widely available, cross-sectional models can be a good choice as first explorations of uncharted territories since cross-sectional data collection is efficient in time, money, and patient impact [60].

Furthermore, a strong assumption of NIRA is that it is possible to precisely target one symptom in a clinical environment. It has been suggested that the 'fat fingers' of psychologists do not account for this 'surgical precision' necessary for symptom-specific interventions due to the interrelatedness of symptoms [61]. For example, treatment interventions aiming to decrease the 'depressed mood' symptom of a patient may focus on changing maladaptive thought patterns [37]. Intervening on these thoughts is likely to affect related symptoms such as 'loss of interest' directly. Thus, changes in symptom activity would not result from alterations in the activation probability of one symptom, which propel to related symptoms, but originate from simultaneous changes in multiple symptoms at the same time. It has even been questioned whether psychiatric symptoms are distinguishable entities at an ontological level, on which distinct interventions can be administered [62]. Some argue that mental states are too overlapping in essence to be considered suitable intervention targets [63]. In other words, the interdependence of symptoms implies their inseparability, rendering it impossible to separate unique contributions of symptoms [64]. In the current paper, we assume that when we intervene on one symptom parameter, all other parameters remain the same. However, we do not study the precise effect of one symptom on another specific symptom, but simulate many data points until the model is converged to its equilibrium state to compute the overall sum score after one intervention. In this way, we consider the interrelatedness of symptoms instead of detangling all possible effects of symptom-specific interventions. Still, it remains an open research 
question whether these symptom-specific interventions have the same effects in an empirical or clinical setting.

Lastly, it is complicated to determine the optimal or empirically realistic way to represent interventions in network models. The magnitude of the computed intervention from NIRA affects the effect size of the projected influences. Weaker interventions lead to more minor projected changes in symptom activity levels. Future research could focus on the different options to represent symptom-specific influences in psychopathology networks. One interesting idea has been proposed by Kruis et al. (in preparation), who adhere different values to the symptom variables in the Ising model. A symptom with a solid projected influence, such as insomnia [65], could be given the binary states $X=\{0,3)$, while a symptom with weak projected influence could be given the values $X=\{0,1)$. In this case, the insomnia variable will have a stronger influence on the dynamics of the model than other symptoms.

Until more research focuses on the empirical validity of intervention studies from the context of psychological networks, the optimal representation of interventions in symptom networks remains an open question. However, we hope the presented technique will be a helpful addition to the methodological toolbox for studying the projected dynamics of symptom networks. In this way, computational models and techniques could aid in improving clinical practices and treatment effectiveness. 


\section{References}

[1] T. F. Blanken et al., "The role of stabilizing and communicating symptoms given overlapping communities in psychopathology networks," Sci. Rep., vol. 8, no. 1, pp. 18, Dec. 2018, doi: 10.1038/s41598-018-24224-2.

[2] D. Borsboom and A. O. J. Cramer, "Network Analysis: An Integrative Approach to the Structure of Psychopathology," Annu. Rev. Clin. Psychol., vol. 9, no. 1, pp. 91-121, Mar. 2013, doi: 10.1146/annurev-clinpsy-050212-185608.

[3] A. M. Chekroud, R. Gueorguieva, H. M. Krumholz, M. H. Trivedi, J. H. Krystal, and G. McCarthy, "Reevaluating the efficacy and predictability of antidepressant treatments: A symptom clustering approach," JAMA Psychiatry, vol. 74, no. 4, pp. 370-378, Apr. 2017, doi: 10.1001/jamapsychiatry.2017.0025.

[4] L. Boschloo et al., "The symptom-specific efficacy of antidepressant medication vs. cognitive behavioral therapy in the treatment of depression: results from an individual patient data meta-analysis," World Psychiatry, vol. 18, no. 2, pp. 183-191, Jun. 2019, doi: 10.1002/wps.20630.

[5] M. J. Dugas and R. Ladouceur, "Treatment of GAD: Targeting intolerance of uncertainty in two types of worry," Behav. Modif., vol. 24, no. 5, pp. 635-657, Jul. 2000, doi: 10.1177/0145445500245002.

[6] C. R. DeCou, K. A. Comtois, and S. J. Landes, "Dialectical Behavior Therapy Is Effective for the Treatment of Suicidal Behavior: A Meta-Analysis," Behav. Ther., vol. 50, no. 1, pp. 60-72, Jan. 2019, doi: 10.1016/j.beth.2018.03.009.

[7] T. M. Lincoln and E. Peters, "A systematic review and discussion of symptom specific cognitive behavioural approaches to delusions and hallucinations," Schizophr. Res., vol. 203, pp. 66-79, Jan. 2019, doi: 10.1016/j.schres.2017.12.014.

[8] E. Bekhuis et al., "Symptom-Specific Effects of Psychotherapy versus Combined Therapy in the Treatment of Mild to Moderate Depression: A Network Approach," Psychother. Psychosom., vol. 87, no. 2, pp. 121-123, Mar. 2018, doi: $10.1159 / 000486793$.

[9] G. Meinlschmidt et al., "Smartphone-based psychotherapeutic micro-interventions to improve mood in a real-world setting," Front. Psychol., vol. 7, no. JUL, p. 1112, Jul. 2016, doi: 10.3389/fpsyg.2016.01112.

[10] D. Borsboom, "A network theory of mental disorders," World Psychiatry, vol. 16, no. 1, pp. 5-13, Feb. 2017, doi: 10.1002/wps.20375.

[11] A. O. J. Cramer et al., "Major Depression as a Complex Dynamic System," PLoS One, vol. 11, no. 12, p. e0167490, Dec. 2016, doi: 10.1371/journal.pone.0167490.

[12] C. Beard et al., "Network analysis of depression and anxiety symptom relationships in a psychiatric sample," Psychol. Med., vol. 46, no. 16, pp. 3359-3369, Dec. 2016, doi: 10.1017/S0033291716002300.

[13] C. Armour, E. I. Fried, and M. Olff, "European Journal of Psychotraumatology PTSD symptomics: network analyses in the field of psychotraumatology," 2017, doi: 10.1080/20008198.2017.1398003.

[14] A.-M. Isvoranu, C. D. van Borkulo, L.-L. Boyette, J. T. W. Wigman, C. H. Vinkers, and D. Borsboom, "A Network Approach to Psychosis: Pathways Between Childhood Trauma and Psychotic Symptoms,” Schizophr. Bull., vol. 43, no. 1, pp. 187-196, Jan. 2017, doi: 10.1093/schbul/sbw055.

[15] M. K. Deserno, D. Borsboom, S. Begeer, and H. M. Geurts, "Relating ASD symptoms to well-being: moving across different construct levels," 2017, doi: 10.1017/S0033291717002616.

[16] D. Borsboom et al., "The small world of psychopathology," PLoS One, vol. 6, no. 11, 
p. e27407, Feb. 2011, doi: 10.1002/wps.20375.

[17] S. Epskamp, A. O. J. Cramer, L. J. Waldorp, V. D. Schmittmann, and D. Borsboom, "Qgraph: Network visualizations of relationships in psychometric data," J. Stat. Softw., vol. 48, no. 4, pp. 1-18, 2012, Accessed: Apr. 13, 2020. [Online]. Available: http://www.jstatsoft.org/.

[18] M. Marsman et al., "An Introduction to Network Psychometrics: Relating Ising Network Models to Item Response Theory Models," Multivariate Behav. Res., vol. 53, no. 1, pp. 15-35, 2018, doi: 10.1080/00273171.2017.1379379.

[19] S. Epskamp and E. I. Fried, "A tutorial on regularized partial correlation networks," Psychol. Methods, vol. 23, no. 4, pp. 617-634, Dec. 2018, doi: 10.1037/met0000167.

[20] S. Epskamp, D. Borsboom, and E. I. Fried, "Estimating psychological networks and their accuracy: A tutorial paper," Behav. Res. Methods, vol. 50, no. 1, pp. 195-212, Feb. 2018, doi: 10.3758/s13428-017-0862-1.

[21] S. Epskamp, L. J. Waldorp, R. Mõttus, and D. Borsboom, "The Gaussian Graphical Model in Cross-Sectional and Time-Series Data," Multivariate Behav. Res., vol. 53, no. 4, pp. 453-480, 2018, doi: 10.1080/00273171.2018.1454823.

[22] C. D. van Borkulo, D. Borsboom, and S. Epskamp, "A new method for constructing networks from binary data," Sci.Rep., 2014, Accessed: Apr. 13, 2020. [Online]. Available: https://www.nature.com/articles/srep05918.

[23] T. R. Spiller, O. Levi, Y. Neria, B. Suarez-Jimenez, Y. Bar-Haim, and A. Lazarov, "On the validity of the centrality hypothesis in cross-sectional between-subject networks of psychopathology," BMC Med., vol. 18, no. 1, p. 297, Oct. 2020, doi: 10.1186/s12916020-01740-5.

[24] M. Newman, Networks: An Introduction. Oxford University Press, 2010.

[25] A. O. J. Cramer, L. J. Waldorp, H. L. J. van der Maas, and D. Borsboom, "Comorbidity: A network perspective," Behavioral and Brain Sciences, vol. 33, no. 23. Cambridge University Press, pp. 137-150, 2010, doi: 10.1017/S0140525X09991567.

[26] D. Robinaugh, R. H. A. Hoekstra, E. R. Toner, and D. Borsboom, "The network approach to psychopathology: a review of the literature 2008-2018 and an agenda for future research," Psychol. Med., vol. 50, no. 3, pp. 353-366, Feb. 2020, doi: 10.1017/S0033291719003404.

[27] J. Stochl, E. Soneson, A. P. Wagner, G. M. Khandaker, I. Goodyer, and P. B. Jones, "Identifying key targets for interventions to improve psychological wellbeing: replicable results from four UK cohorts," Psychol. Med., vol. 49, pp. 2389-2396, 2018, doi: $10.1017 / \mathrm{S} 0033291718003288$.

[28] E. I. Fried et al., "Replicability and Generalizability of Posttraumatic Stress Disorder (PTSD) Networks: A Cross-Cultural Multisite Study of PTSD Symptoms in Four Trauma Patient Samples.," Clin. Psychol. Sci. a J. Assoc. Psychol. Sci., vol. 6, no. 3, pp. 335-351, May 2018, doi: 10.1177/2167702617745092.

[29] L. F. Bringmann et al., "What Do Centrality Measures Measure in Psychological Networks?," J. Abnorm. Psychol., 2019, doi: 10.1037/abn0000446.

[30] F. Dablander and M. Hinne, "Node centrality measures are a poor substitute for causal inference," Sci.Rep., vol. 9, no. 1, pp. 1-13, Dec. 2019, doi: 10.1038/s41598-01943033-9.

[31] M. N. Hallquist, A. G. C. Wright, and P. C. M. Molenaar, "Problems with Centrality Measures in Psychopathology Symptom Networks: Why Network Psychometrics Cannot Escape Psychometric Theory," Multivariate Behav. Res., pp. 1-25, Aug. 2019, doi: 10.1080/00273171.2019.1640103.

[32] D. Castro et al., "The Differential Role of Central and Bridge Symptoms in 
Deactivating Psychopathological Networks," Front. Psychol., vol. 10, p. 2448, Nov. 2019, doi: 10.3389/fpsyg.2019.02448.

[33] T. L. Rodebaugh et al., "Does centrality in a cross-sectional network suggest intervention targets for social anxiety disorder?," J. Consult. Clin. Psychol., vol. 86, no. 10, pp. 831-844, Oct. 2018, doi: 10.1037/ccp0000336.

[34] T. R. Henry, D. J. Robinaugh, and E. I. Fried, "On the control of psychological networks," PsyArXiv, pp. 1-30, 2020, doi: 10.31234/osf.io/7vpz2.

[35] J. M. B. Haslbeck, O. Ryan, D. J. Robinaugh, L. J. Waldorp, and D. Borsboom, "Modeling Psychopathology: From Data Models to Formal Theories," 2019.

[36] D. Robinaugh, A. J. Millner, and R. J. McNally, "Identifying highly influential nodes in the complicated grief network," J. Abnorm. Psychol., vol. 125, no. 6, pp. 747-757, Aug. 2016, doi: 10.1037/abn0000181.

[37] J. Burger et al., "Bridging the gap between complexity science and clinical practice by formalizing idiographic theories: a computational model of functional analysis," $B M C$ Med., vol. 18, no. 99, 2020, doi: 10.1186/s12916-020-01558-1.

[38] J. Barth et al., "Comparative Efficacy of Seven Psychotherapeutic Interventions for Patients with Depression: A Network Meta-Analysis," Focus (Madison)., vol. 14, no. 2, pp. 229-243, Apr. 2016, doi: 10.1176/appi.focus.140201.

[39] E. Ising, "Beitrag zur theorie des ferromagnetismus," Zeitschrift für Phys., vol. 31, no. 1, pp. 253-258, 1925.

[40] C. D. van Borkulo, S. Epskamp, and A. Robitzsch, "IsingFit: Fitting Ising models using the eLasso method," $R$ Packag. version 0.2., 2014.

[41] S. Epskamp, "IsingSampler: Sampling Methods and Distribution Functions for the Ising Model." 2020.

[42] A. F. Danvers, R. Wundrack, and M. Mehl, "Equilibria in Personality States: A Conceptual Primer for Dynamics in Personality States," Eur. J. Pers., vol. 34, no. 6, pp. 999-1016, Nov. 2020, doi: 10.1002/per.2239.

[43] J. P. Nilmeier, G. E. Crooks, D. D. L. Minh, and J. D. Chodera, "Nonequilibrium candidate Monte Carlo is an efficient tool for equilibrium simulation," Proc. Natl. Acad. Sci. U. S. A., vol. 108, no. 45, p. 9665, Nov. 2011, doi: 10.1073/pnas.1106094108.

[44] J. Dalege, D. Borsboom, F. van Harreveld, and H. L. J. van der Maas, "Network Analysis on Attitudes: A Brief Tutorial.," Soc. Psychol. Personal. Sci., vol. 8, no. 5, pp. 528-537, Jul. 2017, doi: 10.1177/1948550617709827.

[45] R. S. Steinberg, A. M., Brymer, M. J., Decker, K. B., \& Pynoos, "The University of California at Los Angeles Post-traumatic Stress Disorder Reaction Index," Curr. Psychiatry Rep., vol. 6, pp. 96-100, 2004.

[46] L. Solá, M. Romance, R. Criado, J. Flores, A. García del Amo, and S. Boccaletti, "Eigenvector centrality of nodes in multiplex networks," Chaos, vol. 23, no. 3, p. 033131, Jul. 2013, doi: 10.1063/1.4818544.

[47] J. M. B. Haslbeck and L. J. Waldorp, "mgm: Estimating Time-Varying Mixed Graphical Models in High-Dimensional Data," J. Stat. Softw., vol. 93, no. 8, Oct. 2020, Accessed: Apr. 13, 2020. [Online]. Available: http://arxiv.org/abs/1510.06871.

[48] E. I. Fried, "Theories and Models: What They Are, What They Are for, and What They Are About," Psychological Inquiry, vol. 31, no. 4. Routledge, pp. 336-344, 2020, doi: 10.1080/1047840X.2020.1854011.

[49] O. Guest and A. E. Martin, "How computational modeling can force theory building in psychological science," 2020.

[50] D. Borsboom, H. van der Maas, J. Dalege, R. Kievit, and B. Haig, "Theory Construction Methodology: A practical framework for theory formation in 
psychology," 2020, doi: 10.31234/osf.io/w5tp8.

[51] D. Robinaugh, J. Haslbeck, O. Ryan, E. Fried, and L. Waldorp, "Invisible Hands and Fine Calipers: A Call to Use Formal Theory as a Toolkit for Theory Construction," 2020, doi: 10.31234/osf.io/ugz7y.

[52] G. Schiepek, "A dynamic systems approach to clinical case formulation," Eur.J. Psychol. Assess., vol. 19, no. 3, pp. 175-184, 2003, doi: https://doi.org/10.1027/10155759.19.3.175.

[53] I. van Rooij and G. Baggio, "Theory Before the Test: How to Build HighVerisimilitude Explanatory Theories in Psychological Science.," Perspect. Psychol. Sci., p. 1745691620970604, Jan. 2021, doi: 10.1177/1745691620970604.

[54] P. C. M. Molenaar, "A Manifesto on Psychology as Idiographic Science: Bringing the Person Back Into Scientific Psychology, This Time Forever," Meas. Interdiscip. Res. Perspect., vol. 2, no. 4, pp. 201-218, Oct. 2004, doi: 10.1207/s15366359mea0204_1.

[55] E. L. Hamaker, "Why researchers should think 'within-person': A paradigmatic rationale.," in Handbook of research methods for studying daily life, M. R. Mehl and T. S. Conner, Eds. The Guilford Press, 2012, pp. 43-61.

[56] L. F. Bringmann et al., "A Network Approach to Psychopathology: New Insights into Clinical Longitudinal Data," PLoS One, vol. 8, no. 4, Apr. 2013, doi: 10.1371/journal.pone.0060188.

[57] H. Lütkepohl, A. Staszewska-Bystrova, and P. Winker, "Comparison of methods for constructing joint confidence bands for impulse response functions," Int. J. Forecast., vol. 31, no. 3, pp. 782-798, Jul. 2015, doi: 10.1016/j.ijforecast.2013.08.003.

[58] X. Yang, N. Ram, J. P. Lougheed, P. C. M. Molenaar, and T. Hollenstein, “Adolescents' emotion system dynamics: Network-based analysis of physiological and emotional experience," Dev. Psychol., vol. 55, no. 9, pp. 1982-1993, Sep. 2019, doi: 10.1037/dev0000690.

[59] A. Inoue and L. Kilian, "Inference on impulse response functions in structural VAR models," J. Econom., vol. 177, no. 1, pp. 1-13, Nov. 2013, doi: 10.1016/j.jeconom.2013.02.009.

[60] P. E. Spector, "Do Not Cross Me: Optimizing the Use of Cross-Sectional Designs," J. Bus. Psychol., vol. 34, no. 2, pp. 125-137, Apr. 2019, doi: 10.1007/s10869-018-096138.

[61] R. J. McNally, "Network Analysis of Psychopathology: Controversies and Challenges," Annu. Rev. Clin. Psychol., vol. 17, pp. 31-53, 2021, doi: 10.1146/annurev-clinpsy-081219.

[62] N. S. de Boer, L. C. de Bruin, J. J. G. Geurts, and G. Glas, "The Network Theory of Psychiatric Disorders: A Critical Assessment of the Inclusion of Environmental Factors," Front. Psychol., vol. 12, p. 623970, Feb. 2021, doi: 10.3389/fpsyg.2021.623970.

[63] J. Woodward, "Cause and explanation in psychiatry: an interventionist perspective," in Philosophical issues in psychiatry: Explanation, phenomenology and nosology, K. S. Kendler and J. Parnas, Eds. Baltimore: John Hopkins University Press, 2014, pp. 209272.

[64] M. Olthof, F. Hasselman, F. O. Maatman, A. Bosman, and A. Lichtwarck-Aschoff, "Complexity Theory of Psychopathology," doi: 10.31234/OSF.IO/F68EJ.

[65] T. F. Blanken, T. Van Der Zweerde, A. Van Straten, E. J. W. Van Someren, D. Borsboom, and J. Lancee, "Introducing Network Intervention Analysis to Investigate Sequential, Symptom-Specific Treatment Effects: A Demonstration in Co-Occurring Insomnia and Depression," Psychother. Psychosom., vol. 88, no. 1, pp. 52-54, Mar. 2019, doi: 10.1159/000495045. 
[66] J. M. B. Haslbeck, S. Epskamp, M. Marsman, and L. J. Waldorp, "Interpreting the Ising Model: The Input Matters," Multivariate Behav. Res., 2020, doi:

10.1080/00273171.2020.1730150. 


\section{TABLES}

Table 1

Title: PTSD Symptoms and their corresponding domains

\begin{tabular}{lll}
\hline Domain & Symptom & Node \\
\hline Intrusion & Intrusive thoughts & B1 \\
\cline { 2 - 3 } & Nightmares & B2 \\
\cline { 2 - 3 } & Flashbacks & B3 \\
\cline { 2 - 3 } & Emotional reactivity & B4 \\
\cline { 2 - 3 } & Physical reactivity & B5 \\
\hline Avoidance & Avoidance of thoughts & C1 \\
\cline { 2 - 3 } & Avoidance of reminders & C2 \\
\cline { 2 - 3 } & Amnesia for aspects & C3 \\
\cline { 2 - 3 } & Loss of interest & C4 \\
\cline { 2 - 3 } & Feeling distant & C5 \\
\cline { 2 - 3 } & Feeling numb & C6 \\
\cline { 2 - 3 } & Foreshortened future & C7 \\
\hline Arousal & Sleep disturbance & D1 \\
\cline { 2 - 3 } & Irritability & D2 \\
\cline { 2 - 3 } & Difficulty concentrating & D3 \\
\cline { 2 - 3 } & Hypervigilance & D4 \\
\cline { 2 - 3 } & Exaggerated startle & D5 \\
\hline
\end{tabular}




\section{PREPRINT WITHOUT PEER-REVIEW}

Table 2

Title: Comparison Between Strength Centrality and NIRA Interventions

\begin{tabular}{|c|c|c|c|c|c|}
\hline \multirow{2}{*}{$\begin{array}{l}\text { Centrality } \\
\text { Strength }\end{array}$} & & \multicolumn{4}{|c|}{ NIRA } \\
\hline & & \multicolumn{2}{|c|}{ Alleviating Interventions } & \multicolumn{2}{|c|}{ Aggravating Interventions } \\
\hline 1 & B4 & 1.94 & B1 & 1.76 & $\mathrm{C} 7$ \\
\hline 0.99 & $\mathrm{C} 6$ & 1.94 & C6 & 1.75 & B3 \\
\hline 0.98 & B3 & 1.93 & B4 & 1.61 & B5 \\
\hline 0.86 & $\mathrm{~B} 1$ & 1.87 & D2 & 1.57 & $\mathrm{C} 4$ \\
\hline 0.83 & B5 & 1.78 & D3 & 1.47 & $\mathrm{C} 5$ \\
\hline 0.43 & $\mathrm{C} 4$ & 1.7 & D1 & 1.24 & $\mathrm{C} 2$ \\
\hline 0.34 & $\mathrm{C} 2$ & 1.65 & B2 & 1.11 & D1 \\
\hline 0.34 & $\mathrm{C} 5$ & 1.63 & $\mathrm{C} 1$ & 1.04 & $\mathrm{~B} 2$ \\
\hline 0.31 & $\mathrm{D} 2$ & 1.6 & $\mathrm{C} 2$ & 1.04 & D5 \\
\hline 0.08 & $\mathrm{D} 1$ & 1.46 & $\mathrm{C} 5$ & 0.82 & D3 \\
\hline 0.02 & D3 & 1.45 & D5 & 0.82 & D2 \\
\hline-0.24 & $\mathrm{C} 7$ & 1.42 & B5 & 0.78 & B1 \\
\hline-0.3 & $\mathrm{C} 1$ & 1.38 & $\mathrm{C} 4$ & 0.68 & $\mathrm{C} 1$ \\
\hline-0.42 & $\mathrm{~B} 2$ & 1.22 & B3 & 0.68 & C6 \\
\hline-0.84 & D5 & 1.17 & D4 & 0.6 & B4 \\
\hline-1.68 & $\mathrm{D} 4$ & 1.12 & $\mathrm{C} 3$ & 0.53 & $\mathrm{C} 3$ \\
\hline-2.69 & $\mathrm{C} 3$ & 0.76 & $\mathrm{C} 7$ & 0.28 & D4 \\
\hline
\end{tabular}




\section{Appendix A: Sensitivity Analyses with Smaller Interventions}

We conducted a sensitivity analysis to study how NIRA results change when interventions are represented by altering the threshold parameters with one standard deviation from the thresholds' distribution - instead of two standard deviations.

Results indicate that the projected effects from NIRA are smaller compared to the results reported in our study, as can be expected from the weaker intervention. However, the projected effects from NIRA still differ over the distinct nodes. Potential propelling effects diminish, as node-specific interventions decrease the network's sum score with a maximum of one. In other words, the symptom-specific intervention is projected to deactivate the targeted symptom, but no neighboring symptoms.

\section{Figure 9}

Projected Effects from Weaker Alleviating NIRA Interventions

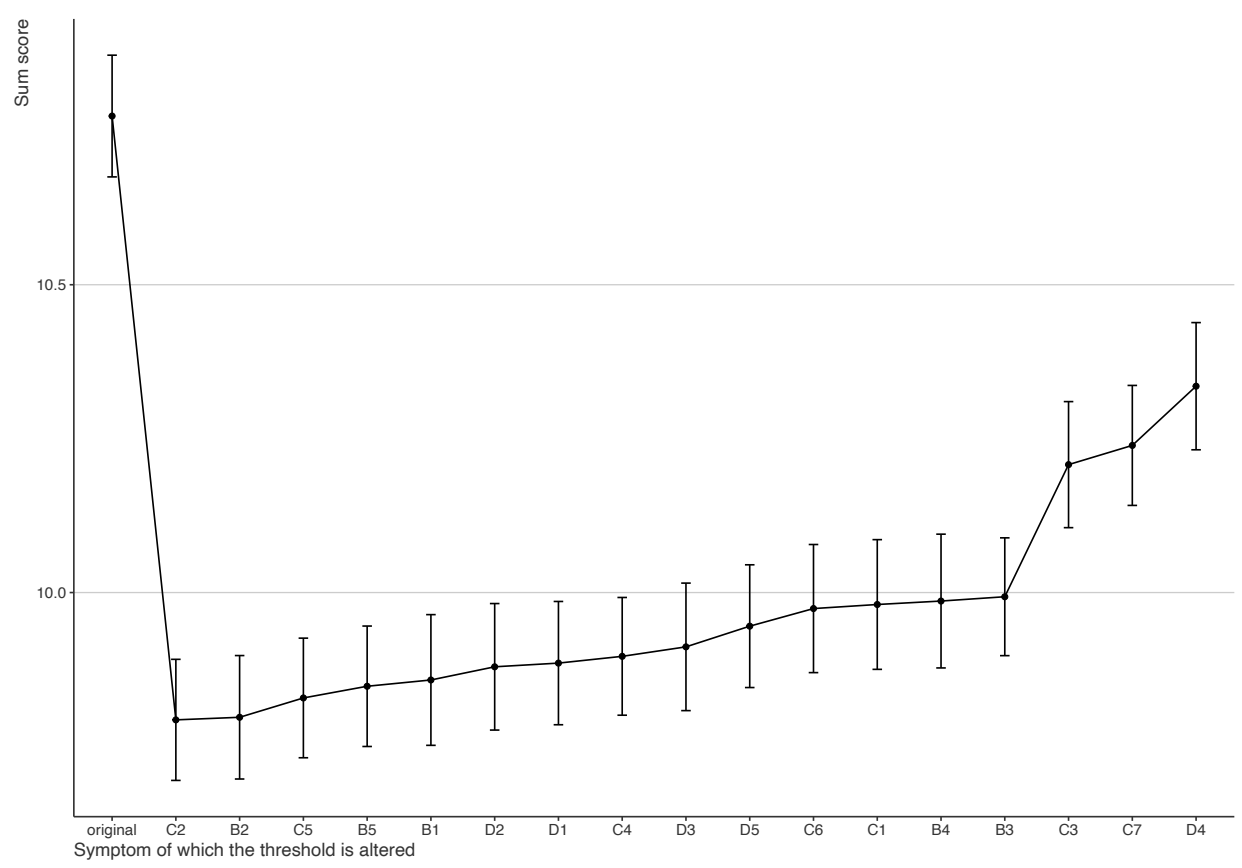

Note. Projected effects from alleviating NIRA interventions when interventions are weaker than the ones reported in the main study. 


\section{Figure 10}

Projected Effects from Weaker Aggravating NIRA Interventions

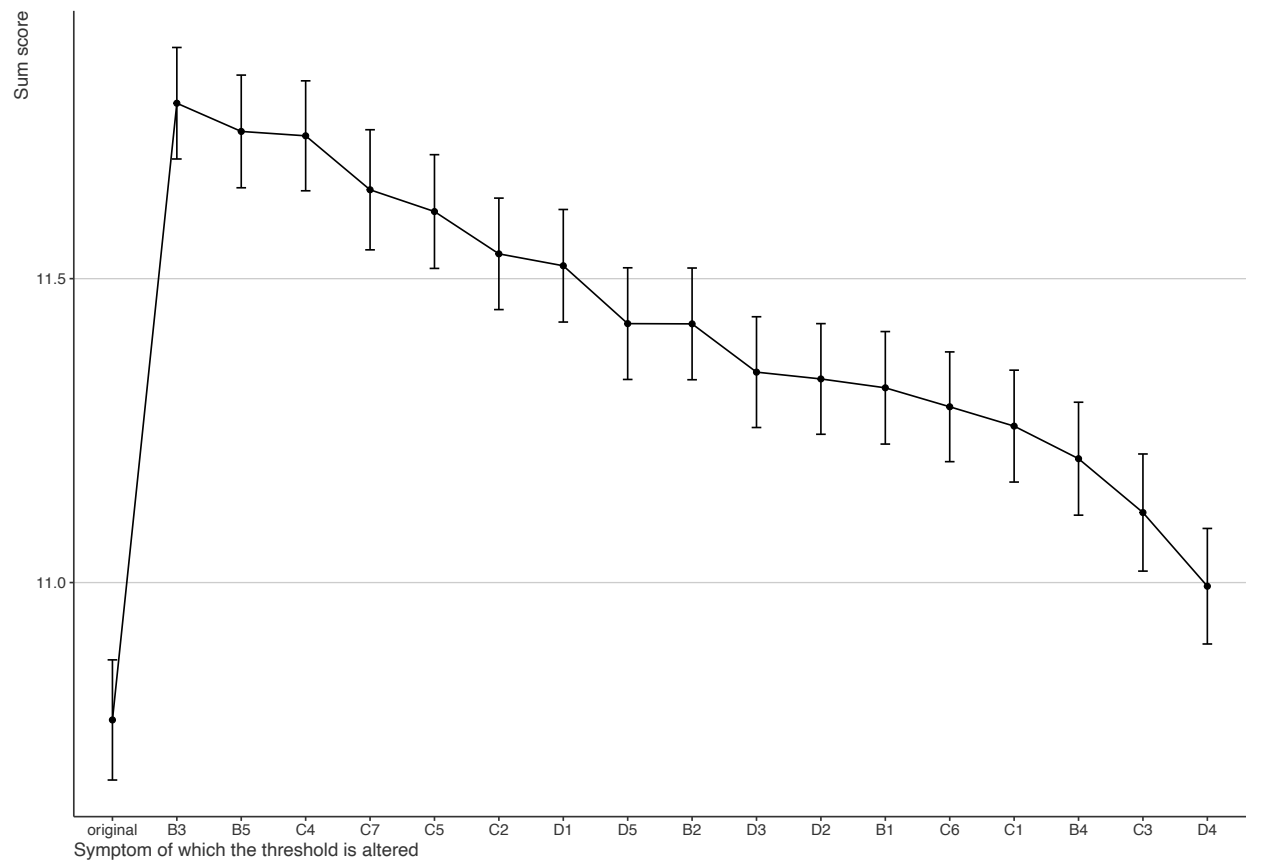

Note. Projected effects from aggravating NIRA interventions when interventions are weaker than the ones reported in the main study. 


\section{Appendix B - Stability Analyses of NIRA using Bootstrapping}

We tested the stability of NIRA outcomes using bootstrapping [20]. The test is conducted using the same principles as the algorithm itself, however, as a starting point, a sample size of 100.000 observations is used. The input network for this test remains the PTSD network used in the empirical illustration of the paper. The steps of the algorithm previously described in section 2.1 are followed (except for the increased sample size) until the sum score distributions are yielded. The test stores the 11 distributions of 100.000 sum scores and now selects 90.000 observations from the original 100.000. For this sample size the sum score distributions are also stored, and the average Pearson correlation between these sum score distribution and the sum score distribution of 100.000 observations is computed. These steps are repeated with sample sizes of $80.000,70.000,60.000,50.000,40.000,30.000$, $20.000,10.000,5000,1000$ and 500. In every step, more cases are dropped from the original 100.000 observations. Therefore, the test technique can best be described as a case dropping bootstrap, which was inspired by the bootnet R package (Epskamp \& Fried, 2019), in which a similar technique is presented.

Figure 11 shows the test results. Each point represents the average Pearson correlation between the sum score distributions of 100.000 observations and the sum score distributions of the corresponding number of observations shown on the x-axis. The grey area surrounding the points represents the $95 \%$ confidence interval given the correlation and the relevant sample size. From this figure a drop can be seen in the correlations with a cut-point after the sample sizes under 5000. On the other hand, all computed correlations are above 0.97 , which is extremely high. To be on the safe side, we use the algorithm with a default sample size of 5000. However, even with smaller sample sizes, the algorithm has proven to be a reliable and stable method.

\section{Figure 11}

Stability Analyses of the NIRA using Bootstrapping

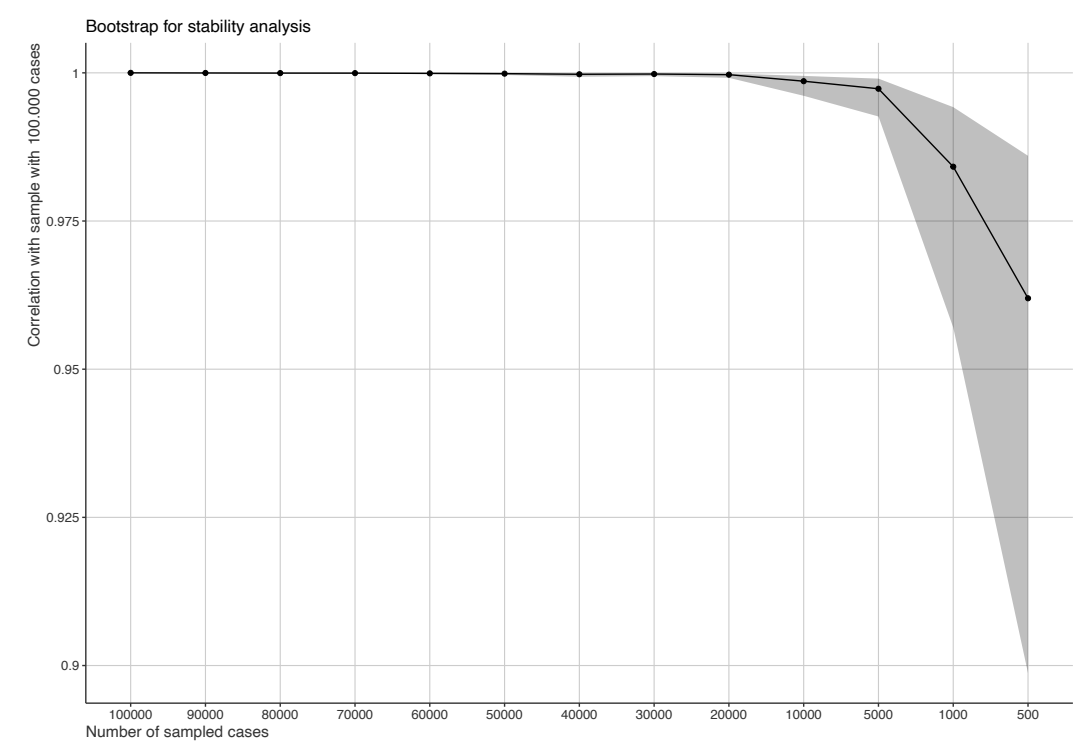

